\title{
Connected Corticospinal Sites Show Enhanced Tuning Similarity at the Onset of Voluntary Action
}

\author{
Yuval Yanai, ${ }^{1}$ Nofya Adamit, ${ }^{1}$ Ran Harel, ${ }^{1,2}$ Zvi Israel, ${ }^{3}$ and Yifat Prut ${ }^{1}$ \\ ${ }^{1}$ The Hebrew University, Hadassah Medical School and the Interdisciplinary Center for Neural Computation, Jerusalem 91120, Israel, ${ }^{2}$ Department of \\ Neurosurgery, Sheba Medical Center, Tel Aviv 52621, Israel, and 3Department of Neurosurgery, Hadassah Hospital, Jerusalem 91120, Israel
}

Corticospinal (CS) pathways provide the structural foundation for executing voluntary movements. Although the anatomy of these pathways is well explored, little is known about spinal decoding of parametric information transmitted via this route during voluntary movements. We addressed this question by simultaneously recording cortical and spinal activity in primates performing an isometric wrist task with multiple targets while measuring CS interactions. Single-pulse cortical stimulation effectively produced a short-latency (presumably monosynaptic) spinal response and thus revealed functionally connected CS sites. Spinal and cortical neurons recorded from connected CS sites showed alignment of directional-torque tuning that peaked at torque onset, consistent with the enhanced cortical drive active during this period. This increased tuning similarity was accompanied by an increased trial-to-trial covariability of firing. Whereas functional CS interactions were dynamic, the efficacy of cortical stimulation was unaffected by the motor state. These results suggest that around the onset of motor action there is a period of facilitated information transfer during which cortical command has greater efficacy in recruiting spinal neurons with matching tuning properties. Dynamic alignment of response properties may form the basis for a spinal readout mechanism of descending motor commands in which directional-torque is a parameter that is preserved across interacting CS sites.

Key words: motor cortex; spinal cord; corticospinal connectivity; directional tuning; noise correlation; motor control

\section{Introduction}

During motor action, a cortical command reaches the spinal circuitry and, according to some yet unknown principle, recruits appropriate neurons to generate the required pattern of muscle activation. Output units of the motor cortex [pyramidal tract neurons (Evarts, 1969; Cheney and Fetz, 1980)] and spinal interneurons (Maier et al., 1998a; Perlmutter et al., 1998; Prut and Fetz, 1999) have been shown independently to fire in relation to directional force during the preparation and execution of movements. Different models have attempted to account for the unique contribution of each of these representations to motor control(Fetz et al., 1996; Georgopoulos, 1996; Bizzi et al., 2000; Todorov, 2000; Kakei et al., 2003). However, studying these representations independently is somewhat inadequate, as motor output is shaped via continuous interactions between motor cortex and the spinal cord.

Corticospinal (CS) pathways provide the motor cortex with both direct and indirect access to muscles. Currently, there is conflicting evidence regarding the relative importance of each of these routes in producing voluntary movements. The direct path,

Received Jan. 26, 2007; revised Sept. 10, 2007; accepted Sept. 11, 2007.

This work was support by Israel Science Foundation Grants ISF-555/01 and ISF-1355/05, and by the generous support of the Baruch Foundation. We thank Steve Perlmutter, Yaacov Ritov, Moshe Abeles, and Eilon Vaadia for useful comments and help.

Correspondence should be addressed to Yifat Prut, Department of Physiology, The Hebrew University-Hadassah Medical School, P.0. Box 12272, Jerusalem 91120, Israel. E-mail: yifatpr@ekmd.huji.ac.il.

DOI:10.1523/JNEUROSCI.3127-07.2007

Copyright $\odot 2007$ Society for Neuroscience ～0270-6474/07/2712349-09\$15.00/0 made up of corticomotoneuronal (CM) cells (Fetz and Cheney, 1980; Lemon et al., 1986) seems to imply a low level control of motor cortex on motor actions (Fetz and Cheney, 1980; Scott and Kalaska, 1995; Todorov, 2000) that does not require the specific involvement of spinal interneurons (INs) in actively processing cortical commands (Lemon et al., 2004). However, anatomical evidence (Kuypers and Brinkman, 1970) tends to indicate that most of the cortically derived motor commands are delegated to muscles via segmental INs. This latter, indirect pathway, is more suggestive of a high level of cortical representation of information which is further processed by spinal systems (Tresch et al., 1999; Bizzi et al., 2000). Nevertheless, the indirect pathway is considered to be weak (Jankowska et al., 1975; Baldissera et al., 1981), particularly in primates compared with lower mammals (Maier et al., 1998b; Nakajima et al., 2000) presumably because of increased inhibitory control (Alstermark et al., 1999; Isa et al., 2006).

In addition to activating motor circuitries, descending cortical commands modulate spinal reflexes (Stein and Capaday, 1988; McCrea, 1992) and thus affect sensory integration in a timedependent manner (Rudomin and Schmidt, 1999; Hultborn, 2001; Seki et al., 2003). This process can greatly alter the balance among converging inputs at the spinal level, and hence modify their relative efficacy. The net impact of these diverse interactions between motor cortex and spinal circuitry and the extent to which cortical input uses segmental circuitry to further process motor commands during voluntary movements remains unclear.

We addressed these questions directly by identifying presum- 


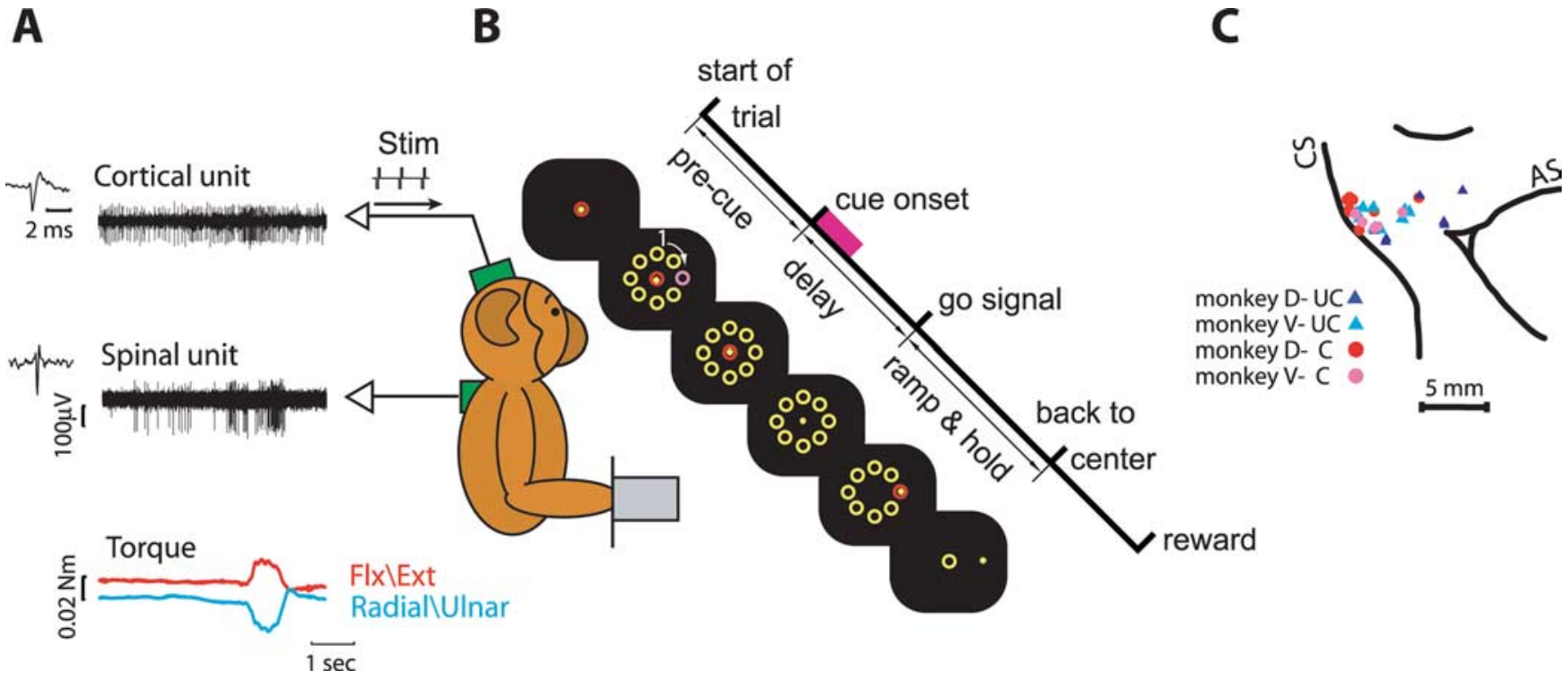

Figure 1. Behavioral task and experimental setup. $\boldsymbol{A}$, Recording configuration. Monkeys sat in front of a computer screen and controlled an on-screen cursor by applying isometric twodimensional wrist torque. Single-unit recordings were made from motor cortex and cervical spinal cord simultaneously. $\boldsymbol{B}$, Sequence of events composing a single trial. The marked events include trial onset, onset of visual cue, go signal, back-to-center signal, and reward. Precue period, delay period, and ramp and hold period are shown as well. Time at which target is filled (cue on) is marked by a magenta square. C, Cortical penetration map of the two monkeys superimposed. Connected $(\boldsymbol{O})$ and unconnected $(\boldsymbol{\Delta})$ sites are shown for monkey D (red and blue) and monkey V (magenta and cyan). Some sites were found in a single session; hence, symbol location was slightly jittered for visibility. CS, Central sulcus; AS, arcuate sulcus; UC, unconnected; C, connected; Flx, flexion; Ext, extension.

ably connected cortical and spinal neurons in behaving primates, and measuring their target-related response. Our working hypothesis was that any similarity in response properties that uniquely characterizes connected cortical and spinal sites (compared with unconnected sites) should point to the parametric information transmitted in the CS route. Using this approach we found that directly connected CS sites were readily identified using cortical microstimulation, suggesting an extensive and robust convergence of descending pathways on spinal circuitry. Cortical and spinal neurons recorded from connected sites expressed tuning alignment at movement onset accompanied by an increased rate of covariation. The results illustrate some dynamic properties of the interactions between motor cortex and spinal cord.

\footnotetext{
Materials and Methods

Animals and behavioral task

Two monkeys (Maccaca fascicularis) performed an isometric twodimensional (2D) wrist task with an instructed delay period (Fig. 1). During task performance, the monkey held its hand in either a pronation or supination position and controlled a cursor on a computer screen by applying a 2D isometric torque at the wrist (flexion/extension and radial/ ulnar). A trial was initiated by the appearance of a central target. The monkey positioned the cursor inside the target by generating zero torque for a rest period (500-600 ms). Then, eight peripheral targets appeared uniformly distributed around the center target at a fixed distance, defining the onset of a delay period. One of these peripheral targets was presented in a distinct color for $500 \mathrm{~ms}$ (cue). The disappearance of the central target (monkey D, 850-1200 ms; monkey V, 1300-1700 ms after cue onset) served as a "go" signal. The monkey then had to acquire the previously filled target by generating an isometric torque in the appropriate direction, and to keep the cursor within the target box for an active torque period ( $350-750 \mathrm{~ms})$. Subsequently, the peripheral targets disappeared and the central target reappeared. The monkey returned to the rest position and received a reward, after which the screen went blank for $1000-1500 \mathrm{~ms}$ and a new trial started.
}

\section{Recording sessions}

Details of the recording technique are described previously (Prut and Perlmutter, 2003a). A cortical chamber $(27 \times 27 \mathrm{~mm})$ was implanted above the motor cortex and the location of arm-related primary motor cortex was mapped using a train of stimulating pulses ( $50 \mathrm{~ms}$ of biphasic stimulation given at $300 \mathrm{~Hz}$ with intensity $\leq 60 \mu \mathrm{A}$ ). Subsequently, a spinal chamber was implanted above the cervical spinal cord (C6-T1). Extracellular single-unit activity (gain, $10 \mathrm{~K}$; bandpass filter, 300-6000 $\mathrm{Hz}$ ) was recorded simultaneously from motor cortex and spinal interneurons located at intermediate lamina while the monkey performed the task. To ensure isolation quality we used off-line spike sorting (AlphaSort; Alpha Omega, Nazareth, Israel) based on principal-component analysis to extract spike times from the compound signal. Only neurons that expressed stable firing across trials were taken for further analysis.

In each recording session, after sufficient data were collected, biphasic (0.2 ms each phase) stimulation pulses were given at $2-3 \mathrm{~Hz}$ (with an intensity of up to $250 \mu \mathrm{A}$ ). After recordings were completed one monkey (monkey D) was deeply anesthetized (ketamine, pentobarbital, $30 \mathrm{mg} /$ $\mathrm{kg}$ ) and pins were inserted into known coordinates of the cortical implant. The animal was then killed with pentobarbital sodium $(50 \mathrm{mg} / \mathrm{kg}$, i.v.) and perfused with $4 \%$ paraformaldehyde. Cortical locations of penetrations relative to anatomical landmarks were subsequently reconstructed. All surgical and animal handling procedures were according to the National Institutes of Health Guide for the Care and Use of Laboratory Animals (1996), complied with Israeli law, and were approved by the Ethics Committee of the Hebrew University. Animal care was supervised by the veterinarian staff of the Hebrew University.

\section{Data analysis}

Identifying connected CS sites. To identify spinal responses to cortical stimulation we first removed the mean stimulation artifact (computed for each stimulation session at a given amplitude) from the single sweep signal. Then, data were digitally bandpass-filtered (eighth-order Butterworth filter, $3-6 \mathrm{kHz}$ ) to remove any low-frequency component which could contain a field effect (see supplemental Fig. 1, available at www. jneurosci.org as supplemental material) and rectified. This processing is equivalent to extraction of a multiunit signal from the compound extracellular signal (Brosch et al., 1997). Averaging the rectified signal is sim- 
ilar in principle to the widely used method of averaging muscle activity around spike timing or applied stimulations (Fetz and Cheney, 1980; Cheney and Fetz, 1985; Bennett and Lemon, 1996; Perlmutter et al., 1998) and reflects a similar purpose: identifying the multiunit response to cortical stimulation. We thus refer to the mean response amplitude in a given time window (either averaged over repeated stimuli or in a single sweep) as a measure of multiunit activity.

To determine corticospinal functional connectivity we used a pairedsample Wilcoxon signed rank test to compare multiunit activity in a time window 2-8 ms after the cortical stimulus onset to a baseline window -10 to $-4 \mathrm{~ms}$ before cortical stimulus. This was done for a stimulus amplitude of $150 \mu \mathrm{A}$ as this stimulation amplitude was common to all tested sites. Cortical and spinal sites were classified as functionally connected if the test reached a significance level of 0.0014 ( 0.05 divided by 36 , the total number of sites).

Quantifying spinal response. Once connected sites were identified we quantified the evoked effect as follows. We expanded the post stimulus inspection window to span 1.3 to $10 \mathrm{~ms}$. The lower bound was set to the earliest expected post stimulus effect (Fetz and Cheney, 1980). At each site and stimulus amplitude we aligned the processed (see above) single sweeps on stimulus onset-time and averaged them. We then computed the mean and SD of the averaged trace in the baseline window $(-10$ to $-4 \mathrm{~ms}$ before stimulus onset). In the inspection window we looked for all occurrences of sustained activity ( $>0.5 \mathrm{~ms}$, equivalent to 13 samples) above the mean plus two SDs of the baseline. Note that for a given average trace, multiple periods of sustained activity can be found. The onset and offset times of such sustained activity were defined to be the first preceding or subsequent bin below the mean plus one SD respectively. Response magnitude was the integral of the activity above the mean baseline in all epochs of sustained activity divided by the baseline SD. Onset latency was defined as the start time of the earliest occurrence of sustained activity.

To test whether the response magnitude was epoch-dependent we first classified single stimulus traces according to the behavioral epochs in which they occurred: rest (500 ms preceding cue onset), delay (cue onset to torque onset), ramp (torque onset to hold onset), and hold (hold onset to hold offset). Torque onset and offset were determined, for each trial by an ad hoc method based on a threshold crossing of the time derivative of the exerted torque. For each trace we computed the single trace response magnitude as the difference between the multiunit activity in the post stimulus window $(+2 . .8 \mathrm{~ms})$ and the multiunit activity in the pre stimulus window ( -10 to $-4 \mathrm{~ms})$. Finally, for each site and stimulus amplitude, we ran a Kruskal-Wallis test to compare single trace response magnitudes from different epochs.

Signal correlation analysis. For each neuron, we computed the targetrelated signal (i.e., tuning curve) during ramp and hold (200 ms before torque onset to $1000 \mathrm{~ms}$ after torque onset, Fig. 3C). This tuning curve was simply the mean firing rate of the neuron while moving toward each target across trial repetitions. For each pair of simultaneously recorded cortical and spinal units, we computed the signal cross-correlation by calculating the correlation coefficient between their target-related signals (Lee et al., 1998). We then $z$-transformed the correlation coefficients (Sokal and Rohlf, 1981) because the distribution of the untransformed values was greatly skewed. Normalizing these distributions allowed us to use a $t$ test to compare the correlation obtained for connected versus unconnected sites. We used an additional form of interneuronal similarity measure based on the target-independent temporal profile of the firing rate. For each neuron we computed in each direction the perievent time histogram (PETH) during ramp and hold as defined above. The temporal profile was obtained by counting spikes in a $300 \mathrm{~ms}$ sliding window that was moved in $50 \mathrm{~ms}$ steps. We then averaged these histograms across targets to obtain a target-independent temporal profile. From here, the similarity between PETH of cortical and spinal neurons was measured as described above for the signal correlation analysis.

Time-resolved signal-correlation analysis. For each neuron, timedependent relationships between firing rate and target position were estimated. We aligned single trials on the time of torque onset and computed single-trial spike counts in a sliding window spanning $300 \mathrm{~ms}$ and moving in $50 \mathrm{~ms}$ steps. For each time window we computed the signal of the neuron by computing the tuning curve (as defined above) during this specific time window. This measure was termed the "instantaneous signal," defined here as the signal computed for a time window spanning $300 \mathrm{~ms}$. Hereafter, the word "instantaneous" will be used to refer to a single $300 \mathrm{~ms}$ time window.

For each pair of simultaneously recorded cortical and spinal units, we computed the time-resolved signal cross-correlation by calculating the correlation coefficient between their instantaneous signals throughout the trial and at varying interneuronal time lags $(-500$ to $+500 \mathrm{~ms}$ in 50 ms steps) (see Fig. 4). We then $z$-transformed the correlation coefficients. To evaluate the significance of the results we compared, bin-by-bin, the two sets of signal correlation matrices obtained from connected and unconnected sites (two-sample $t$ test). The result was a matrix of $p$ values and the significance threshold was set at $0.05 / N$, where $N$ is the number of bins in the matrix used here to correct for multiple comparisons (Bonferoni correction). For presentation purposes, the $p$ value matrix was converted into a Surprise matrix defined as $-\log (p / 1-p)$ and was smoothed by convolving it with a two-dimensional Gaussian $\left(\sigma_{\mathrm{x}}=\sigma_{\mathrm{y}}=\right.$ $100 \mathrm{~ms}$ ).

Time-resolved noise correlation analysis. A noise correlation matrix was computed in a similar manner to the signal correlation. For a single trial and a specific time window we computed the instantaneous noise (i.e., the noise in a $300 \mathrm{~ms}$ time window) by subtracting the average firing rate from the instantaneous firing rate and dividing by the SD of the firing rate. Both the average and the SD of the firing rate were computed for trials with the same target during that time window.

The time-resolved noise cross-correlation was then measured as the correlation coefficient between the instantaneous noise of cortical and spinal neurons throughout the trial and at varying time delays ( -500 to $+500 \mathrm{~ms}$ in $50 \mathrm{~ms}$ steps) (Fig. 4). Only pairs of neurons which were recorded simultaneously for at least 10 trials qualified for this analysis.

Note that estimating noise correlation is equivalent to studying crosscorrelation of firing rates between neurons, after removing the targetrelated modulation of firing from both rate estimates. Nevertheless, the relatively large time window used in this study prevents us from detecting fine temporal interactions (i.e., spike-time correlation) between single neurons; rather, we were limited to detecting rate-based interactions, namely, changes in the firing rate (computed using a time bin of $300 \mathrm{~ms}$ ) of one neuron in temporal relations to such changes expressed by a second neuron.

Comparison between connected sites and unconnected sites was performed in a similar manner to the signal correlation with the exception that here the significance threshold was set at 0.05 . This difference in significance threshold reflects the fact that the noise correlation is a second-order statistic (measuring cofluctuations around a mean level) which tends to yield weaker values than signal correlation. Furthermore, the noise-correlation analysis was performed after the signal-correlation analysis pointed to a region of interest in the $2 \mathrm{D}$ matrix. Because we focused on a smaller portion of this matrix, a more lenient significance threshold was warranted.

\section{Results}

We studied the functional relationships of 165 pairs of cortical and spinal neurons recorded from 15 connected and 21 unconnected sites.

\section{Identifying functionally connected corticospinal sites}

To estimate the information transmitted through descending commands we sought to compare the similarity in response properties of cortical and spinal neurons that are functionally connected with those that are functionally independent. The first step in this analysis was to identify connected CS sites. Finding pairs of directly connected spinal and cortical neurons in the intact animal is practically impossible. Instead, we delivered biphasic single pulse electrical stimulation in the motor cortex while recording spinal activity. We found that these pulses produced a clear and significant spinal multiunit response (Fig. 2A) in more than a third of the recorded corticospinal pairs ( 7 of 21 
and 8 of 15 of the sites in monkeys $\mathrm{V}$ and $\mathrm{D}$, respectively). On average, response magnitude increased as stimulation amplitude increased, although inconsistencies were observed (Fig. $2 G$ ). These inconsistencies could partially be related to mixed excitatory and inhibitory effects (Fig. 2D), which may express different amplitude relations. Subsequently, the amplitude-related increase in the excitatory response might have been curtailed by the appearance of an inhibitory response at higher stimulation amplitudes. The mean response latency for all stimulation amplitudes in all sites was $2.8 \mathrm{~ms}$. The earliest expected disynaptic response was 0.7-0.9 ms after the earliest observed monosynaptic response (Maier et al., 1998b; Perlmutter et al., 1998). In each site we considered the fastest response onset time across all stimulation amplitudes to estimate the synaptic distance. The fastest response in monkey D occurred at $2.0 \mathrm{~ms}$. Four of eight of the sites had fastest response onsets $<2.7 \mathrm{~ms}$, whereas the other four sites had fastest response onsets $<3.2$ ms. In monkey $\mathrm{V}$, the fastest response onset occurred at $1.6 \mathrm{~ms}$. Three of seven sites had fastest response onsets $<2.3 \mathrm{~ms}$ whereas the other four sites had fastest response onsets $<3.0 \mathrm{~ms}$. Thus in almost half of the cases the spinal response occurred at a short latency, which can be explained by a monosynaptic (but not disynaptic) connection. For the remaining sites, the results are more ambiguous because they can be defined as either slow monosynaptic or fast disynaptic connections.

The presence or absence of spinal response to cortical stimulation (see Materials and Methods) was used to classify pairs of cortical and spinal neurons into those recorded from connected or unconnected sites, respectively.

\section{Similar response properties in functionally connected sites}

Next, we explored the functional correlates of connected CS sites by estimating the consistency in response properties between simultaneously recorded pairs of cortical and spinal neurons.

Similarity between tuning properties of cells is often quantified by the signal correlation (Averbeck and Lee, 2004), which measures cosimilarity in response properties between neurons. We first tested two forms of interneuronal response similarity during the ramp and hold periods $(-200$ to $+1000 \mathrm{~ms}$ relative to torque onset). The first was based on the target-related signal, which was measured as the average firing rate in the test window for each target (thus producing an eight-dimensional tuning curve). The correlation between these signals computed for pairs of cortical and spinal neurons recorded from connected sites was significantly higher than the correlation values obtained for neurons recorded from unconnected sites (Fig. 3A). The second mea-
$\mathrm{E}$

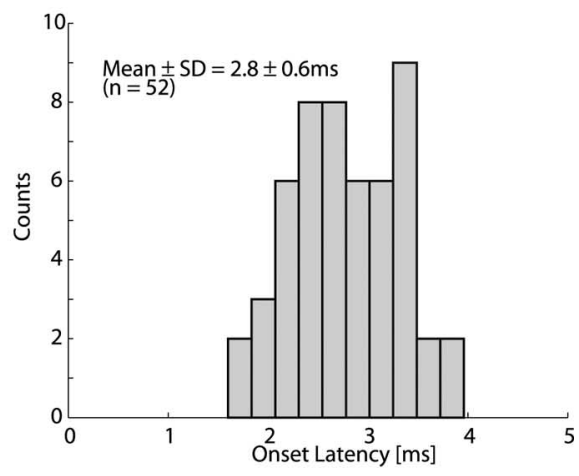

$\mathrm{F}$

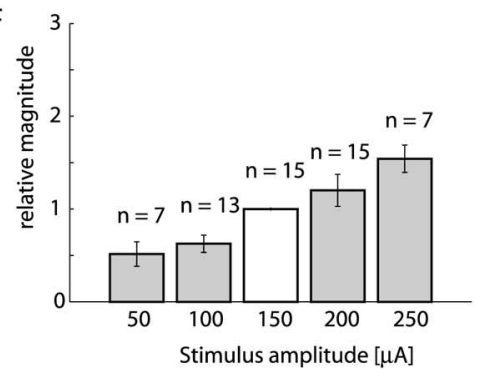

G
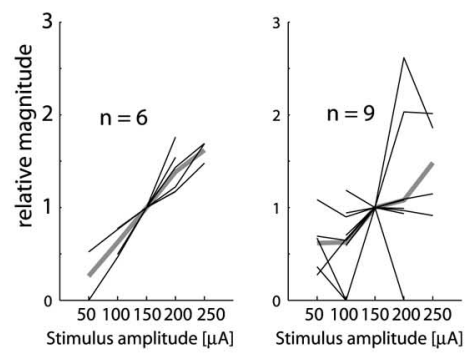

Figure 2. Recruitment of spinal neurons by single cortical pulses. A, Aligning single traces of spinal recordings on stimulus onset time reveals a clear neuronal response ( 5 traces randomly chosen from the applied stimulations). A stimulus artifact appears preprocessed traces over 202 stimulus repetitions. Area filled with dark gray was used to compare response magnitude across sites and stimulation intensities. D, Additionally observed response types included double-peak response (top), excitation followed by stimulation locations and amplitudes. $\boldsymbol{F}$, Relationship between response magnitude and stimulation amplitude. To eliminate the amplitude in single sites (thin black lines) and their average (thick gray lines). Monotonically increasing relations were found in 6 of 15 sites (left), but not for the other 9 of 15 sites (right). However, even in the latter case, the mean response magnitude across sites increased monotonically with stimulus amplitude.

sure we used to quantify response similarity was based on the target-independent temporal profile of the firing rate during ramp and hold which is the average perievent time histogram (aligned on torque onset time) across all targets. The correlation between these firing profiles computed for neurons recorded from connected sites were similar to that found for pairs recorded from unconnected sites (Fig. 3B).

\section{Time-resolved signal and noise correlation}

We found that target-related information is likely to be transmitted between functionally connected sites. However, many cells in our study exhibited modulations in their target-related firing over the course of the trial (Fig. 3C,D). We therefore expanded the search for target-related signal correlations between cortical and spinal neurons and considered the signal correlation as a 

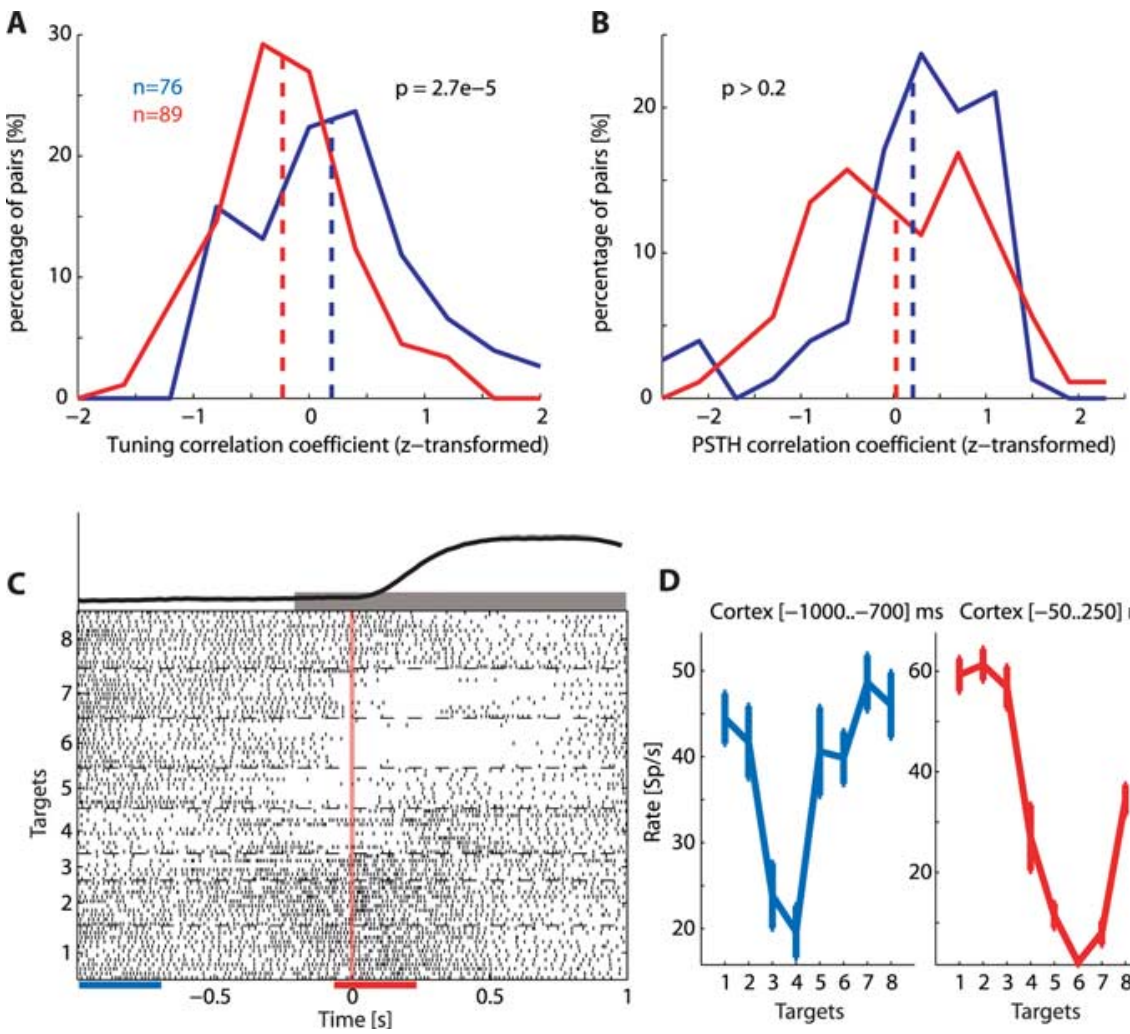

D

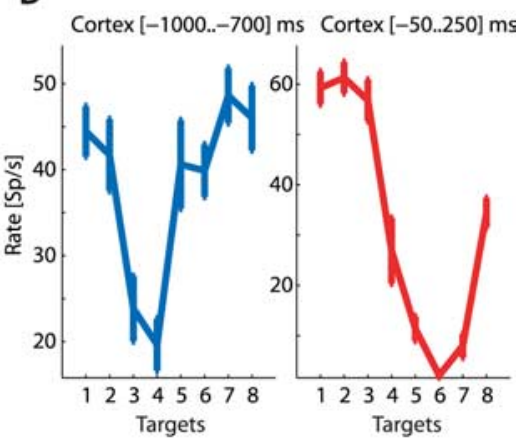

Figure 3. Signal correlation depends on functional connectivity. A, Distribution of z-transformed correlation coefficients between tuning curves of cortical and spinal pairs of neurons recorded in connected sites (blue) and unconnected sites (red). For each neuron the tuning curve was computed in a time window $(-200$ to +1000$)$ ms around torque onset. $\boldsymbol{B}$, Distribution of $z$-transformed correlation coefficients between temporal profiles of corticospinal pairs recorded in connected sites (blue) and unconnected sites (red). For each neuron the temporal profile was computed during the ramp and hold period by averaging the target specific temporal profiles across all targets. A target-specific temporal profile is a smoothed perievent-time histogram (using a 300 ms sliding window that was moved in 50 ms steps). C, A raster display of a cortical neuron aligned on torque onset and sorted by targets. The gray bar above the raster display indicates the ramp and hold period. Visual inspection suggests that during this time window as well as during the delay the target-related signal changes considerably. Blue and red bars below the raster display represent two sample windows selected to demonstrate this point. Torque profile, averaged across trials in all targets is shown above raster plot. $\boldsymbol{D}$, Tuning curves computed in the two sample windows. Note that the preferred target (i.e., the target for which neuronal firing was maximal) as well as the level of firing changed between the two time windows.

trices from connected sites bin-by-bin against all matrices obtained from unconnected sites to identify statistically significant bins (two sample $t$ test, $p<0.05 /$ number-of-bins, delineated within white borders in Fig. 5C).

The results show that tuning-similarity computed for connected CS sites (Fig. 5A, left) transiently increased before torque onset and before hold onset, namely at points of transition between stable and dynamic torque. In contrast, the signal correlation computed for unconnected sites (Fig. 5A, right) expressed a decrease in signal similarity. The relation between signal correlation value and task performance could be assessed by the average torque profile and its variability (Fig. 5B). These plots further show that the difference in signal correlation observed between connected and unconnected sites cannot be accounted for by systematic differences in task performance.

\section{Rate-based correlation between functionally connected CS sites}

Increased tuning similarity does not in itself dictate any model for CS interactions, as tuning similarity could evolve independently. Furthermore, signal correlation cannot provide an estimate of the strength of interactions between single cortical and spinal neurons. To identify functional, rate-based interactions between CS sites we computed the noise-correlation matrices for the same data set using 75 CS pairs recorded from connected sites and 88 pairs recorded from unconnected sites (Fig. 6, supplemental Fig. 3, available at www. jneurosci.org as supplemental material).

dynamic rather than a constant measure of cell activity along the trial. Moreover, the extent of tuning similarity between spinal and cortical neurons may be further affected by the relative cortical-to-spinal time lag used for computing the signal correlation. For example, the time it takes each network to fully exhibit task-related firing (Eytan and Marom, 2006) may suggest that maximal tuning similarity between cortical and spinal neurons should be expected at nonzero time lags. Indeed, substantial latency has been measured between activity of motor cortical neurons and performed motor actions (Moran and Schwartz, 1999). All these temporal effects were captured by the signal correlation matrix which measures time-resolved changes in the instantaneous CS tuning similarity occurring at different CS lags (Fig. 4). We grouped the interactions from 55 or 21 pairs of neurons recorded from eight or seven connected sites and compared the results to 40 or 49 pairs of neurons recorded from 7 or 14 unconnected sites for monkeys $\mathrm{D}$ and $\mathrm{V}$, respectively. The averaged signal correlation matrix expressed a consistent dynamic for both monkeys in connected as well as unconnected sites (supplemental Fig. 2, available at www.jneurosci.org as supplemental material) and the data were therefore combined (Fig. 5). Our null hypothesis was that signal correlation is independent of the CS functional connectivity classification. We therefore tested all ma-
The results show that units recorded from connected sites had a significantly higher noise correlation compared with units recorded from unconnected sites. Increased noise correlation was strongest just before torque onset, parallel to the increase in signal correlation. Moreover, the increased interactions were biased toward positive CS lags, consistent with cortical activity preceding spinal activity.

\section{Evoked CS efficacy is independent of motor context}

Modulation in functional CS interactions could result from variations in the efficacy of the CS transmission line. To study this possibility we compared the magnitude of the spinal response to cortical microstimulation delivered at four different epochs of the task: rest, delay, ramp, and hold (see Materials and Methods), representing different motor contexts. Because the prestimulus baseline level changed across these epochs we estimated the stimulus-induced change in multiunit activity. At each behavioral epoch, we looked at the mean change in multiunit activity between two time windows: -10 to -4 ms before the stimulation and +2 to $+8 \mathrm{~ms}$ after the stimulation. The underlying assumption was that a behavioral epoch for which cortical efficacy is reduced would show a smaller change in multiunit activity. Only 4 of 53 of the site-amplitude combinations showed a significant 
interaction $(p<0.05)$ between change in multiunit activity and behavioral epoch. Even when pooling evoked responses obtained for all recording sites and all stimulation amplitudes (supplemental Fig. 4, available at www.jneurosci.org as supplemental material) no significant differences were observed in the evoked response applied at different epochs.

\section{Discussion}

Previous studies (Bizzi et al., 2000; Todorov, 2000) hypothesized different functional interactions between motor cortex and spinal cord as part of movement control. Our study addresses this issue directly and shows that these two structures readily interact in a robust and dynamic manner, expressing increased efficacy of the cortical command during torque onset. Furthermore, parametric analyses of these interactions show that directional-torque tuning (or its correlates) of neurons is a property that is preserved when information is transmitted from motor cortex to spinal cord and thus can serve as a control signal in the motor system.

We found that single-pulse stimulations applied in the motor cortex can reveal functionally connected CS sites without the need for spatial or temporal summation. This indicates that in behaving primates, CS interactions are substantially stronger than their presumed strength as might be indirectly estimated based on studies performed in reduced preparations (Jankowska et al., 1976; Baldissera et al., 1981), where often large-scale cortical stimulation (Jankowska et al., $1975,1976)$ or stimulation in the pyramidal tract were used. Similar differences in functional efficacy between awake animals and reduced preparation was also reported for CM connectivity (Porter and Lemon, 1993). The frequency of finding connected CS sites reflects both the convergence of an extensive (although not necessarily continuous) cortical area on spinal sites (Jankowska et al., 1976; He et al., 1993) and the fact that in behaving primates the spinal circuitry operates at an elevated level of excitability (Prut and Perlmutter, 2003a).

The stimulation pulses used in our study clearly excite many cortical neurons in the vicinity of the electrode (Tehovnik, 1996). Motor cortical neurons were shown to be spatially organized in a mosaic-like pattern (Jankowska et al., 1975; Amirikian and Georgopoulos, 2003; Ben-Shaul et al., 2003) where neighboring neurons share similar response properties (Lee et al., 1998) and input/output connectivity patterns (Jackson et al., 2003) whereas response properties across patches are unrelated. It is therefore expected that single-pulse stimulation should mostly excite local cells with some degree of homogeneity in their response properties in addition to more remote cells with randomly varying tuning properties. Our findings were consistent with this organizational scheme: in six tracks we encountered both connected and
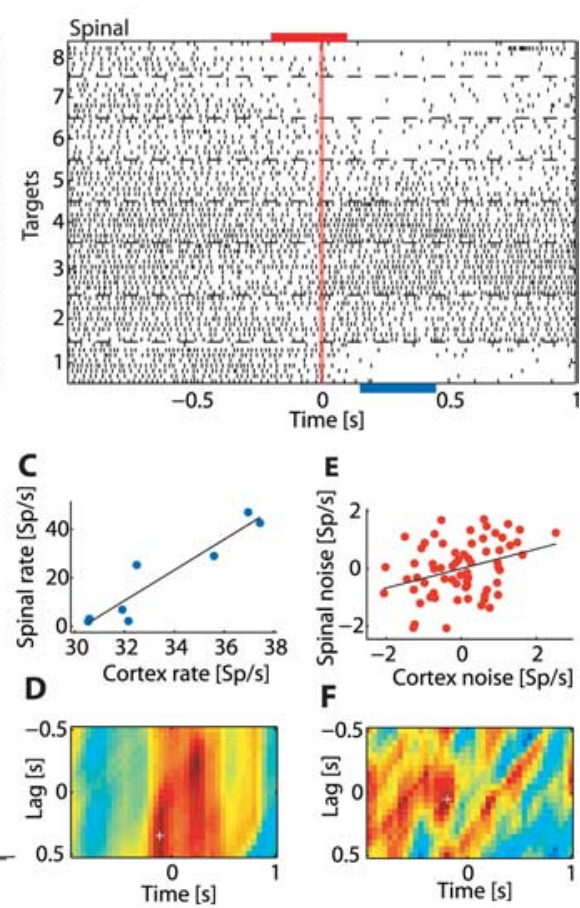

Figure 4. Computing signal and noise correlation matrices for a pair of neurons recorded simultaneously. $\boldsymbol{A}$, Raster displays of The red bars above the raster display represent two different sample windows for which neuronal noise (trial-by-trial deviation time (relative to torque onset) used as the center of the cortical sample window. The $y$-axis is the CS lag between the two sample ( correlation coefficient for different combinations of cortical and spinal sample windows. The layout is similar to the one used for the signal correlation matrix. White cross marks the point that corresponds to the (noise) sample windows shown previously.

unconnected sites, thus further emphasizing that single pulse cortical stimulation can be used to identify CS interactions with a relatively fine spatial resolution.

The structural observation of interacting CS sites had a clear functional correlate in terms of tuning properties: spinal and cortical neurons recorded from connected sites expressed a dynamic alignment of tuning during task performance. The association between these two findings, namely connected CS sites which are uniquely characterized by increased tuning similarity (in contrast to unconnected sites) suggests that tuning alignment is obtained via CS interactions. An alternative (although less intuitive) explanation could be that the increased signal (and noise) is mediated by a specific termination pattern in which a given pathway (e.g., peripheral afferents) preferentially affects matching cortical and spinal sites. However, the latter model cannot account for the rate-based interactions (i.e., noise correlation), which were temporally biased toward lags in which cortical activity precedes spinal activity.

The observed transient nature of CS interaction could potentially reflect the emergence of tuning just before torque onset. In 
A

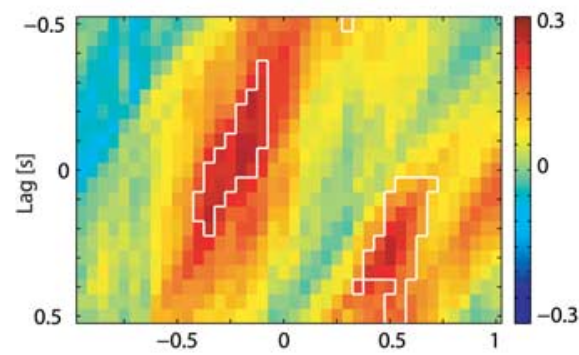

B

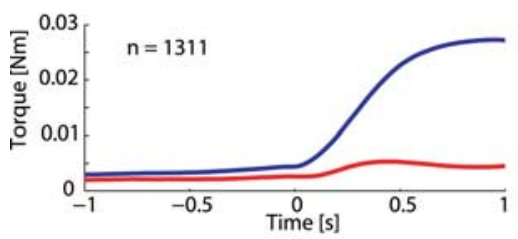

C
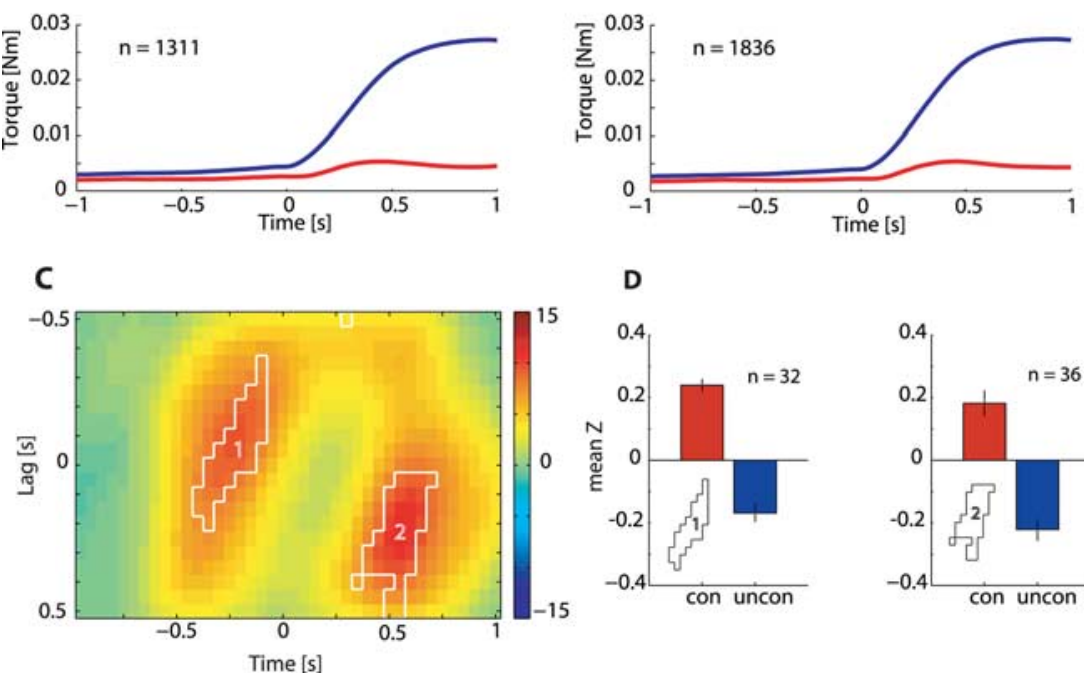

D
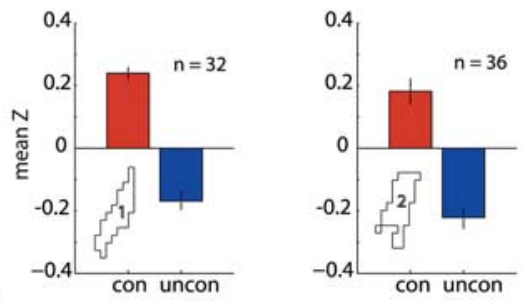

Figure 5. Cortical-to-spinal signal correlation matrices computed for connected and unconnected sites. $A$, Time-resolved signal cross-correlation averaged across all pairs of neurons recorded from functionally connected sites (left, $n=76$; monkey $D$, $n=55$; monkey $\mathrm{V}, n=21$ ) and functionally unconnected sites (right, $n=89$; monkey $\mathrm{D}, n=40$; monkey $\mathrm{V}, n=49$ ). Bins for which correlation values were significantly different between connected and unconnected sites are delineated by a white border. $\boldsymbol{B}$, Mean (blue) and SD (red) of radial torque (distance from center) pooled for all targets in connected sites (left) and unconnected sites (right). Comparing the signal correlation differences to the average torque profile suggests that differences are prominent before torque onset and before hold onset, but not during the early phase of movement. C, Surprise matrix highlights the bins with a significant ( $p<6 \times 10^{-5}$; see Materials and Methods) difference between connected and unconnected pairs. The two significant areas are marked 1 and 2 . D, Bars showing the mean of the $z$-transformed correlation coefficient in area 1 (left) and area 2 (right) for functionally connected sites (red) and functionally unconnected sites (blue). The difference between connected sites and unconnected sites is caused by positive correlations in connected sites as well as negative correlations in unconnected sites.

the data presented here, the pretrigger period used for computing the time-resolved matrices was taken from the postcue delay period activity. In this epoch, the spatial direction of the ensuing movement had already been provided. Previous studies have reported target-related preparatory activity in the motor cortex (Tanji and Evarts, 1976; Thach, 1978; Kubota and Funahashi, 1982) and spinal cord (Prut and Fetz, 1999), although not as robust as movement-related tuning. This may suggest that a nonzero signal correlation is expected at this period, unlike the lack of correlation reported here. Moreover, the dynamic tuning similarity was matched by a trial-by-trial rate comodulation at the same time window. The latter parameter is not affected by neuronal tuning properties and, thus, further emphasizes the inherent dynamic nature of CS interactions. Finally, regardless of the actual source for the dynamic CS interactions, their functional implication is the same: target-related information is transmitted in a preferred time window around torque onset.

Functionally connected cortical and spinal neurons had increased tuning similarity, yet it is difficult to identify the exact parameter transmitted in this manner (e.g., directional torque and/or its derivatives). Part of this difficulty is attributable to our current task design where many of the parameters are colinear. Additional study is required to resolve this ambiguity.

Whereas the increased signal similarity between connected sites seems intuitive, the decrease in signal similarity in unconnected sites appears to be more puzzling. This significant decrease suggests that the population of unconnected CS pairs of neurons does not only contain unrelated cells; rather, it includes a subset of neuronal pairs with opposite directional tuning, which thus contributes negative signal correlation values. This subset of pairs may correspond to an indirect (possibly inhibitory) cortical connection with spinal neurons that have the opposite preferred direction. The comparable frequencies of finding connected and unconnected sites further support this view. This point can be illustrated by using a hypothetical extreme situation: let us assume first that the cortical population is equally divided between agonist and antagonist-related sites; second, a given spinal site receives input from all agonist cortical sites and none from antagonist sites. Therefore, if a cortical stimulation does not evoke a spinal reaction we can expect a negative (agonistantagonist) signal correlation rather than a zero correlation.

Signal correlation analysis revealed tuning-based functional CS interactions. A more direct measure for CS interactions was obtained when measuring the CS noise correlation. Signal-dependent noise correlation was found previously for neighboring motor cortical neurons (Lee et al., 1998), but not for spinal INs (Prut and Perlmutter, 2003b). Here, we show that significantly increased noise correlation can be found for connected sites at lags which are consistent with cortical firing preceding spinal firing. The temporal match between signal and noise correlation may suggest a mechanism in which CS interactions mediate tuning similarity. However, some care should be taken when interpreting these results. Falsified noise correlation was shown to emerge in cases of behavioral variability that was unaccounted for (Brody, 1999; Ben-Shaul et al., 2001). In our data, trial-by-trial variability in motor performance (even for a given target) can potentially contribute to such artificial noise correlation. Nevertheless, the fact that positive noise correlation was found for connected sites compared with unconnected sites, although the motor variability in these two sets of data were essentially the same, indicates that the observed noise correlation reflects true CS interactions. Furthermore, a transient noise correlation was found at times when behavioral variability was minimal, namely just before the aligning event, further supporting the validity of this result.

Spinal response evoked by cortical stimulation was not significantly modulated by the motor context. This result is unexpected based on previous reports showing that corticomuscular interactions are task-dependent, possibly because of changes in 
the excitability of corticospinal (Baker et al., 1995; Lemon et al., 1995; Schubert et al., 1997) and/or spinal (Armstrong and Drew, 1985; Matthews, 1986) systems. Similarly reflex pathways were shown to modulate in a behaviorally dependent manner (Stein and Capaday, 1988; Chen et al., 1998; Zytnicki and Jami, 1998; Zehr and Stein, 1999; Hultborn, 2001), often because of changes in the efficacy of specific pathways.

A possible source for this discrepancy is a relatively weak spinal effect that was masked by a large trial-by-trial variability in the spinal baseline level. Indeed, although the context-dependent impact on the evoked response of muscle and descending volley (Di Lazzaro et al., 1998) was clearly robust, it is hard to predict the magnitude of this effect in the response of the local heterogeneous population of INs that was used here to quantify evoked spinal response. Thus, to observe such an effect it may be necessary to average over much larger number of repeated stimuli in any given epoch. Therefore, our results cannot rule out the existence of such a task-dependent effect, but they can provide some upper bound to its strength.

A model that could account for the dynamic CS interactions uses the predominantly phasic (Cheney and Fetz, 1980) and synchronized (Lee et al., 1998; Baker et al., 2001) firing of M1 neurons known to exist at movement onset. This firing pattern could be translated by descending fibers into a particularly effective recruiting drive for spinal neurons (as was suggested for cortical neurons) (Abeles, 1982; Salinas and Sejnowski, 2000). At segmental levels, the descending command has been reported to be further enhanced through its additional role in mediating presynaptic inhibition of afferent information during movement onset in nonhuman (Rudomin and Schmidt, 1999; Seki et al., 2003) and human (Pierrot-Deseilligny and Meunier, 1998) subjects. This presynaptic inhibition may act to change the balance among converging inputs on spinal neurons by amplifying the relative impact of cortical inputs (which are not subjected to this inhibition) (Nielsen and Petersen, 1994; Jackson et al., 2006). In this model, moderate changes in firing pattern of cortical neurons accompanied by gating of competing pathways greatly alter the availability of spinal neurons to be recruited by cortical commands. Overall, the cortical drive for voluntary movements exerts a paramount, albeit transient impact on spinal activity extending beyond its anatomical volume. The outcome of these interactions is that during motor actions, spinal interneurons contain parametric information related to the exerted directional torque. Thus, in primates performing voluntary movements, the indirect CS pathway, routed via segmental INs, may actively participate in processing the descending cortical command before it reaches the muscles.

\section{References}

Abeles M (1982) Role of the cortical neuron: integrator or coincidence detector? Isr J Med Sci 18:83-92.

Alstermark B, Isa T, Ohki Y, Saito Y (1999) Disynaptic pyramidal excitation in forelimb motoneurons mediated via $\mathrm{C}(3)-\mathrm{C}(4)$ propriospinal neurons in the Macaca fuscata. J Neurophysiol 82:3580-3585.

Amirikian B, Georgopoulos AP (2003) Modular organization of directionally tuned cells in the motor cortex: is there a short-range order? Proc Natl Acad Sci USA 100:12474-12479.

Armstrong DM, Drew T (1985) Forelimb electromyographic responses to motor cortex stimulation during locomotion in the cat. J Physiol (Lond) 367:327-351.

Averbeck BB, Lee D (2004) Coding and transmission of information by neural ensembles. Trends Neurosci 27:225-230.

Baker SN, Olivier E, Lemon RN (1995) Task-related variation in corticospinal output evoked by transcranial magnetic stimulation in the macaque monkey. J Physiol 488:795-801.

Baker SN, Spinks R, Jackson A, Lemon RN (2001) Synchronization in monkey motor cortex during a precision grip task. I. Task-dependent modulation in single-unit synchrony. J Neurophysiol 85:869-885.

Baldissera F, Hultborn H, Illert M (1981) Integration in spinal neuronal systems. In: The nervous system (Brookhart JM, Mountcastle VB, eds), pp 509-595. Bethesda, MD: American Physiological Society.

Bennett KM, Lemon RN (1996) Corticomotoneuronal contribution to the fractionation of muscle activity during precision grip in the monkey. J Neurophysiol 75:1826-1842.

Ben-Shaul Y, Bergman H, Ritov Y, Abeles M (2001) Trial to trial variability in either stimulus or action causes apparent correlation and synchrony in neuronal activity. J Neurosci Methods 111:99-110.

Ben-Shaul Y, Stark E, Asher I, Drori R, Nadasdy Z, Abeles M (2003) Dynamical organization of directional tuning in the primate premotor and primary motor cortex. J Neurophysiol 89:1136-1142.

Bizzi E, Tresch MC, Saltiel P, d'Avella A (2000) New perspectives on spinal motor systems. Nat Rev Neurosci 1:101-108.

Brody CD (1999) Correlations without synchrony. Neural Comput 11:1537-1551.

Brosch M, Bauer R, Eckhorn R (1997) Stimulus-dependent modulations of correlated high-frequency oscillations in cat visual cortex. Cereb Cortex 7:70-76. 
Chen R, Yaseen Z, Cohen LG, Hallett M (1998) Time course of corticospinal excitability in reaction time and self-paced movements. Ann Neurol 44:317-325.

Cheney PD, Fetz EE (1980) Functional classes of primate corticomotoneuronal cells and their relation to active force. J Neurophysiol 44:773-791.

Cheney PD, Fetz EE (1985) Comparable patterns of muscle facilitation evoked by individual corticomotoneuronal (CM) cells and by single intracortical microstimuli in primates: evidence for functional groups of CM cells. J Neurophysiol 53:786-804.

Di Lazzaro V, Restuccia D, Oliviero A, Profice P, Ferrara L, Insola A, Mazzone P, Tonali P, Rothwell JC (1998) Effects of voluntary contraction on descending volleys evoked by transcranial stimulation in conscious humans. J Physiol 508:625-633.

Evarts EV (1969) Activity of pyramidal tract neurons during postural fixation. J Neurophysiol 32:375-385.

Eytan D, Marom S (2006) Dynamics and effective topology underlying synchronization in networks of cortical neurons. J Neurosci 26:8465-8476.

Fetz EE, Cheney PD (1980) Postspike facilitation of forelimb muscle activity by primate corticomotoneuronal cells. J Neurophysiol 44:751-772.

Fetz EE, Perlmutter SI, Maier MA, Flament D, Fortier PA (1996) Response patterns and postspike effects of premotor neurons in cervical spinal cord of behaving monkeys. Can J Physiol Pharmacol 74:531-546.

Georgopoulos AP (1996) On the translation of directional motor cortical commands to activation of muscles via spinal interneuronal systems. Brain Res Cogn Brain Res 3:151-155.

He SQ, Dum RP, Strick PL (1993) Topographic organization of corticospinal projections from the frontal lobe: motor areas on the lateral surface of the hemisphere. J Neurosci 13:952-980.

Hultborn H (2001) State-dependent modulation of sensory feedback. J Physiol (Lond) 533:5-13.

Isa T, Ohki Y, Seki K, Alstermark B (2006) Properties of propriospinal neurons in the $\mathrm{C} 3-\mathrm{C} 4$ segments mediating disynaptic pyramidal excitation to forelimb motoneurons in the macaque monkey. J Neurophysiol 95:3674-3685.

Jackson A, Gee VJ, Baker SN, Lemon RN (2003) Synchrony between neurons with similar muscle fields in monkey motor cortex. Neuron 38:115-125.

Jackson A, Baker SN, Fetz EE (2006) Tests for presynaptic modulation of corticospinal terminals from peripheral afferents and pyramidal tract in the macaque. J Physiol (Lond) 573:107-120.

Jankowska E, Padel Y, Tanaka R (1975) Projections of pyramidal tract cells to alpha-motoneurones innervating hind-limb muscles in the monkey. J Physiol (Lond) 249:637-667.

Jankowska E, Padel Y, Tanaka R (1976) Disynaptic inhibition of spinal motoneurones from the motor cortex in the monkey. J Physiol (Lond) 258:467-487.

Kakei S, Hoffman DS, Strick PL (2003) Sensorimotor transformations in cortical motor areas. Neurosci Res 46:1-10.

Kubota K, Funahashi S (1982) Direction-specific activities of dorsolateral prefrontal and motor cortex pyramidal tract neurons during visual tracking. J Neurophysiol 47:362-376.

Kuypers HG, Brinkman J (1970) Precentral projections to different parts of the spinal intermediate zone in therhesus monkey. Brain Res 24:29-48.

Lee D, Port NL, Kruse W, Georgopoulos AP (1998) Variability and correlated noise in the discharge of neurons in motor and parietal areas of the primate cortex. J Neurosci 18:1161-1170.

Lemon RN, Mantel GW, Muir RB (1986) Corticospinal facilitation of hand muscles during voluntary movement in the conscious monkey. J Physiol (Lond) 381:497-527.

Lemon RN, Johansson RS, Westling G (1995) Corticospinal control during reach, grasp, and precision lift in man. J Neurosci 15:6145-6156.

Lemon RN, Kirkwood PA, Maier MA, Nakajima K, Nathan P (2004) Direct and indirect pathways for corticospinal control of upper limb motoneurons in the primate. Prog Brain Res 143:263-279.

Maier MA, Perlmutter SI, Fetz EE (1998a) Response patterns and force relations of monkey spinal interneurons during active wrist movement. J Neurophysiol 80:2495-2513.
Maier MA, Illert M, Kirkwood PA, Nielsen J, Lemon RN (1998b) Does a C3-C4 propriospinal system transmit corticospinal excitation in the primate? An investigation in the macaque monkey. J Physiol (Lond) 511:191-212.

Matthews PB (1986) Observations on the automatic compensation of reflex gain on varying the pre-existing level of motor discharge in man. J Physiol (Lond) 374:73-90.

McCrea DA (1992) Can sense be made of spinal interneuron circuits? Behav Brain Sci 15:633-643.

Moran DW, Schwartz AB (1999) Motor cortical representation of speed and direction during reaching. J Neurophysiol 82:2676-2692.

Nakajima K, Maier MA, Kirkwood PA, Lemon RN (2000) Striking differences in transmission of corticospinal excitation to upper limb motoneurons in two primate species. J Neurophysiol 84:698-709.

Nielsen J, Petersen N (1994) Is presynaptic inhibition distributed to corticospinal fibres in man? J Physiol (Lond) 477:47-58.

Perlmutter SI, Maier MA, Fetz EE (1998) Activity of spinal interneurons and their effects on forearm muscles during voluntary wrist movements in the monkey. J Neurophysiol 80:2475-2494.

Pierrot-Deseilligny E, Meunier S (1998) Differential control of presynaptic inhibition of Ia terminals during voluntary movement in humans. In: Presynaptic inhibition and neural control (Rudomin P, Romo R, Mendell L, eds), pp 351-365. New York: Oxford UP.

Porter R, Lemon RN (1993) Corticospinal function and voluntary movement. Oxford: Calrendon.

Prut Y, Fetz EE (1999) Primate spinal interneurons show pre-movement instructed delay activity. Nature 401:590-594.

Prut Y, Perlmutter SI (2003a) Firing properties of spinal interneurons during voluntary movement. I. State-dependent regularity of firing. J Neurosci 23:9600-9610.

Prut Y, Perlmutter SI (2003b) Firing properties of spinal interneurons during voluntary movement. II. Interactions between spinal neurons. J Neurosci 23:9611-9619.

Rudomin P, Schmidt RF (1999) Presynaptic inhibition in the vertebrate spinal cord revisited. Exp Brain Res 129:1-37.

Salinas E, Sejnowski TJ (2000) Impact of correlated synaptic input on output firing rate and variability in simple neuronal models. J Neurosci 20:6193-6209.

Schubert M, Curt A, Jensen L, Dietz V (1997) Corticospinal input in human gait: modulation of magnetically evoked motor responses. Exp Brain Res 115:234-246.

Scott SH, Kalaska JF (1995) Changes in motor cortex activity during reaching movements with similar hand paths but different arm postures. J Neurophysiol 73:2563-2567.

Seki K, Perlmutter SI, Fetz EE (2003) Sensory input to primate spinal cord is presynaptically inhibited during voluntary movement. Nat Neurosci 6:1309-1316

Sokal RR, Rohlf FJ (1981) Biometry, Ed 2. New York: W. H. Freeman.

Stein RB, Capaday C (1988) The modulation of human reflexes during functional motor tasks. Trends Neurosci 11:328-332.

Tanji J, Evarts EV (1976) Anticipatory activity of motor cortex neurons in relation to direction of an intended movement. J Neurophysiol 39:1062-1068.

Tehovnik EJ (1996) Electrical stimulation of neural tissue to evoke behavioral responses. J Neurosci Methods 65:1-17.

Thach WT (1978) Correlation of neural discharge with pattern and force of muscular activity, joint position, and direction of intended next movement in motor cortex and cerebellum. J Neurophysiol 41:654-676.

Todorov E (2000) Direct cortical control of muscle activation in voluntary arm movements: a model. Nat Neurosci 3:391-398.

Tresch MC, Saltiel P, Bizzi E (1999) The construction of movement by the spinal cord. Nat Neurosci 2:162-167.

Zehr EP, Stein RB (1999) What functions do reflexes serve during human locomotion? Prog Neurobiol 58:185-205.

Zytnicki D, Jami L (1998) Presynaptic inhibition can act as a filter of input from tendon organs during muscle contraction. In: Presynaptic inhibition and neural control, pp 303-314. Oxford: Oxford UP. 